\title{
Obesity-relationship with vascular dysfunction
}

\begin{abstract}
Obesity is defined as abnormal or excessive fat accumulation that may impair health. Obesity and overweight are considered as a high-income country problem. At least 2.8 million people die each year as a result of being overweight or obese. This review, discusses the relationship between obesity, nutritional determinants and health impacts. Obesity comorbidities include coronary heart disease, hypertension and stroke, certain types of cancer, non-insulin-dependent diabetes mellitus, gallbladder disease, Dyslipidemia, osteoarthritis and gout, and pulmonary diseases, including sleep apnea.

The literature was searched to identify relevant manuscripts, as well as meta-analyses, studying the obesity and vascular dysfunction relationship. Broad search terms were used that related to obesity factors such as physical activity, exercise, diet, nutrition, eating, food, diabetes, weight, etc. The study shows that adipose tissue is an active endocrine organ which plays a central role in lipid and glucose metabolism. It also produces a large number of hormones and cytokines involved in the development of metabolic syndrome, diabetes mellitus, and vascular diseases. Weight reduction and increasing physical activity are effective interventions for improving adipose tissue function.
\end{abstract}

Volume I Issue I - 2014

\author{
Radina Dimitrova,' Valentina Petkova,' Milen \\ Dimitrov, ${ }^{2}$ Vasil Madzharov, ${ }^{3}$ Irina Nikolova, ${ }^{4}$ \\ Elina Petkova,' Kalina Andreevska, ${ }^{3}$ Daniela \\ Grekova, ${ }^{3}$ Stanislav Gueurguiev ${ }^{3}$ \\ 'Department of Social Pharmacy, Medical University, Bulgaria \\ ${ }^{2}$ Department of Pharmaceutical Technology and Biopharmacy, \\ Medical University, Bulgaria \\ ${ }^{3}$ Department of Pharmaceutical sciences, Medical University, \\ Bulgaria \\ ${ }^{4}$ Department of Pharmacology, Pharmacotherapy and Toxicology, \\ Medical University, Bulgaria
}

\begin{abstract}
Correspondence: Valentina Petkova, Department of Social Pharmacy, Medical University, Sofia, Bulgaria, Tel 003599236593 ,
\end{abstract} Fax 003599879874, Email petkovav1972@yahoo.com

Received: July 0I, 2014 | Published: July 29, 2014

Keywords: obesity, vascular dysfunction, risk factors, complications

Abbreviations: BMI, body mass index; WHO, world health organization; AHA, American heart association; TNF- $\alpha$, tumour necrosis factor- $\alpha$; IL-6, interleukin-6; ICAM-1, intercellular adhesion molecule-1; VLDL, very low-density lipoprotein; LDL, low-density lipoprotein; HDL, high density lipoprotein; NEFAs, non-esterified fatty acids; PAI-1, plasminogen activator inhibitor-1; PVAT, perivascular adipose tissue; NO, nitric oxide; RAS, renin-angiotensin-aldosterone system; AGT, angiotensinogen; ACE, angiotensin converting enzyme; HDL-c, high density lipoprotein- cholesterol

\section{Introduction}

Excess visceral fat deposition in the abdominal cavity is a high risk factor for cardiovascular disease, insulin resistance and metabolic syndrome. The prevalence of obesity has been increasing alarmingly and it has now become a global concern causing severe burden on health care systems, with readily available effective therapies or preventive measures in demand. The rich potential of nature to combat obesity and its associated complications (i.e. insulin resistance, vascular dysfunction and adipose tissue abnormalities) has not been fully explored yet, and many newer preventive and/or therapeutic strategies can be obtained from the natural source.

\section{Obesity as a world-wide social problem}

Obesity is a world-wide epidemic considered to be the fifth leading risk for global deaths. Obesity and its associated conditions such as insulin resistance, type 2 diabetes, Dyslipidemia and steatosishepatis, termed as a metabolic syndrome, represent major challenges for basic science and clinical research. Obesity is defined as abnormal or excessive fat accumulation that may impair health. The World Health Organization (WHO) defines obesity with BMI (body mass index):

a. A BMI greater than or equal to 25 is overweight

b. A BMI greater than or equal to 30 is obesity. ${ }^{1}$
Obesity is considered to be the disease of the modern and the urbanization world, the consequences of which may be fatal. Every year millions of people die as a result of being overweight or obese. According to the WHO $44 \%$ of the diabetes burden, $23 \%$ of the ischaemic heart disease burden and between $7 \%$ and $41 \%$ of certain cancer burdens (endometrial, breast, colon) are due to obesity. ${ }^{1}$ Obesity and overweight are considered as a high-income country problem, even though there is a rise in low and middle-income countries. This makes about $10 \%$ of all the population worldwide suffering of obesity. Most worrying is the fact that children even under the age of 5, who live in developing or developed countries, have overweight problems or obesity. ${ }^{1,2}$ Obesity is caused by an energy imbalance between calories consumed and calories expended. The high-fat food consumption and the low physical activity results in excessive fat accumulation, which increases the risk of non-communicable diseases such as:

a. Cardiovascular diseases- mainly heart disorders and stroke- the leading cause of death in 2008

b. Diabetes

c. Musculoskeletal disorders- especially osteoarthritis

d. Some cancers- endometrial, breast, colon ${ }^{1}$

At least 2.8 million people die each year as a result of being overweight or obese. In fact, the mortality increases with the increasing of the BMI. In the WHO European Region, the Eastern Mediterranean Region and the Region of the Americas, over 50\% of women are overweight. The highest prevalence of overweight among infants and young children is in upper-middle-income populations, while the fastest rise in overweight is in the lower-middle-income group. ${ }^{1,3-5}$ In order to maintain good health, individuals need to stay within the range of $18.5-24.9 \mathrm{~kg} / \mathrm{m}^{2}$ BMI. There is increased risk of co-morbidities for BMIs in the range of 25.0 to $29.9 \mathrm{~kg} / \mathrm{m}^{2}$, very high risk of co-morbidities for a BMI greater than $30 \mathrm{~kg} / \mathrm{m}^{2}$. In the year of 
2008 , more than 205 million men and 297 million women worldwide were estimated with the condition of obesity. In high income countries there is almost no difference between the number of men and women suffering of obesity, while in lower-middle-income countries or in upper-middle income countries, obese women are more than double than obese men. ${ }^{6-10}$

A research conducted in the Netherlands, Spain, Sweden and the United Kingdom, have shown an inverse relationship between education and either BMI or obesity among both men and women. Other research shows that obesity has relation with the socioeconomic status of men, women and children in medium - and low-income countries. In 2008 there were 40million pre-school children with weight more than double than the normal at that age. Gaining weight depends on the socioeconomic status in different countries. In the lower and middle- income countries the number of children with obesity rises within the rising of incomes. On the other hand, in highincome countries, such as the United Kingdom and the United States, lower socioeconomic status is associated with a higher prevalence of obesity. ${ }^{11}$ Globalization- consequence of increased urbanization and industrialization, including international trade and shared communications, and the lack of active lifestyle, results in rapidly spreading obesity and turning it into a worldwide problem.

Before 1980 , obesity rates were generally much lower than $10 \%$. Since then, rates have doubled or tripled in many countries and nowadays the database shows that $50 \%$ or more of the population is overweight. $^{12-14}$

In many countries the threat of spreading the obesity and becoming a national problem is rapidly growing and increases many concerns. It is in the leading positions of non-communicable diseases and recently replaces more traditional problems such as under nutrition and infectious diseases as the most significant causes of ill-health. Obesity comorbidities include coronary heart disease, hypertension and stroke, certain types of cancer, non-insulin-dependent diabetes mellitus, gallbladder disease, dyslipidemia, osteoarthritis and gout, and pulmonary diseases, including sleep apnea. The main problem in gaining weight comes from the prevalence intake of energy fats and the lack of active lifestyle. Eating food rich in saturated fats and refined sugar, coupled with the lack of sporting activity, inevitably leads to increasing the body mass index. ${ }^{1,9,15}$

\section{Methodology}

\section{Identification of relevant studies}

The literature was searched to identify relevant manuscripts, as well as meta-analyses. Four electronic databases were searched to identify reviews of relationship between obesity and vascular dysfunction (Google Scholar, Research Gate, EMBASE, PubMed) published until May 2014. Broad search terms were used that related to obesity factors such as physical activity, exercise, diet, nutrition, eating, food, diabetes, weight, etc). Criteria for inclusion of papers were that they reported a systematic review or meta-analysis and were published in English. Reviews of intervention studies were also included. Identified papers were first screened for inclusion based on the title, followed by a screening of the abstract, and then the full text. The reference lists of included papers were screened for further relevant studies. Any duplicate papers were removed.

\section{Quality rating}

The included reviews were rated for methodological quality based on the following criteria a. Inclusion of an explicit and clear statement regarding subject of the study.

b. Comprehensiveness of the methodology.

c. Clarity of the description of inclusion and exclusion criteria.

d. The quality assessment included ratings of at least two of the following aspects: study design, sampling, outcome measures and follow-up assessment.

\section{Results}

\section{Health effects of obesity}

Obesity is a fast growing problem that is reaching epidemic proportions worldwide, and is associated with an increased risk of premature death. The consequences for the cardiovascular system may be irreversible. This includes: stroke, congestive heart failure, myocardial infarction and cardiovascular death. The American Heart Association (AHA) has classified obesity as a 'major, modifiable risk factor' for coronary heart disease. ${ }^{2,15,16}$ Except the cardiovascular consequences, obesity has also a major influence in other systems in the organism. Adipose tissue is an active endocrine and paracrine organ that releases a large number of cytokines and bioactive mediators, such as leptin, adiponectin, interleukin- 6 (IL-6) and tumour necrosis factor- $\alpha$ (TNF- $\alpha$ ), that influence not only body weight homeostasis but also insulin resistance, diabetes, lipid levels, tension, coagulation, fibrinolysis, inflammation and atherosclerosis. ${ }^{17-23}$

Obesity is also associated with increased levels of endothelial cell products such as intercellular adhesion molecule-1 (ICAM-1). Cigarette smoking may also influence cytokines: it causes a dosedependent increase in plasma ICAM-1 and decrease in adiponectin levels, thereby inducing endothelial dysfunction. It is not known yet what substance of the cigarette smoke causes this problems. Smoking may also increase levels of tumor necrosis factor- $\alpha$ (TNF- $\alpha$ ), a powerful cytokine that might decrease adiponectin levels and induce insulin resistance. Lower adiponectin levels found in chronic smokers confirm earlier findings that chronic smokers are insulin resistant. ${ }^{22,23}$

Dyslipidemia in obesity is characterized by increased levels of very low-density lipoprotein (VLDL) cholesterol, triacylglycerols and total cholesterol, an increase in small dense LDL particles, and lower high density lipoprotein (HDL) cholesterol levels. Thus, the chance of developing cardiovascular disease will rise. If it is used a treatment with statins, the risk of the formation of coronary plaquesin people with high body weight, is reduced. As it is known increasing the levels of LDL and lowering the levels of HDL higher the risk of cardio-vascular problems, including atherosclerosis. This happens when the LDL particles are not taken up by the LDL-receptors, but by macrophage scavenger receptors. The artery wall thickens as a result of invasion and accumulation of white blood cells, macrophages and dead cells, including cholesterol (http://en.wikipedia.org/ wiki/Cholesterol) and triglycerides (http://en.wikipedia.org/wiki/ Triglyceride). This is promoted by low-density lipoproteins (http:// en.wikipedia.org/wiki/Low-density_lipoprotein), without adequate removal of fats and cholesterol from the macrophages by functional high-density lipoproteins (http://en.wikipedia.org/wiki/Highdensity_lipoprotein). The hepatic overproduction of VLDL is due to hepatic steatosis - a condition which occurs as a result of obesity. The excessive levels of VLDL are crucial for the organism. Insulin resistance in the liver, muscles and adipose tissue leads to an inability to suppress hepatic glucose production, impaired glucose uptake and oxidation, and inability to suppress release of non-Esterified fatty 
acids (NEFAs) from adipose tissue. ${ }^{16,24}$ ApoB-100 degradation plays role in the regulation of VLDL secretion, but in this condition of insulin resistance the level of ApoB-100 is decreased. The levels of triglycerides are also increased due to reduction in lipoprotein lipase activity. ${ }^{16}$

\section{Vascular and adipose tissue dysfunction in obesity}

Obesity is characterized by excessive accumulation of adipose tissue (fat)- a highly dynamic endocrine organ, secreting a large number of bioactive substances that controls insulin sensitivity, energy metabolism, immune responses and cardiovascular homeostasis. Adipose-secreted factors modulate endothelial regeneration, vascular tone and cardiac contractility. The secretion of these vasoactive molecules from adipose tissues leads to endothelial dysfunction, which is characterized by impaired vasodilatation and increased vasoconstriction. Thus the insulin resistance gets worse and in adipose tissue start an inflammation. ${ }^{21,23,25}$ The adipose tissue has a central role in lipid and glucose metabolism and produces a large number of hormones and cytokines, e.g. angiotensinogen, tumour necrosis factor-a (TNF-a), interleukin-6 (IL-6), adiponectin, leptin, and plasminogen activator inhibitor-1 (PAI-1). A major driver of adipose tissue function is the quantity of visceral fat. ${ }^{4}$

There is a close anatomical and functional relationship between adipose tissue and blood vessels. Adipose tissue is highly vascularized and rich supply of blood flow is essential for its metabolic functions and expansion. Vascular endothelium also secretes many factors to regulate adipogenesis and adipose tissue remodeling. ${ }^{21}$ Almost all blood vessels are surrounded by Perivascular adipose tissue (PVAT), which regulates vascular function by producing a large number of "vasocrine" molecules. PVAT has anti-contractile effects by releasing vasorelaxants, such as: adipocyte-derived relaxation factors and adiponectin. They induce relaxation of blood vessels. ${ }^{21}$ In obesity PVAT becomes very inflamed and changes its function into secretion of vasoconstriction factors, such as: the major components of renin-angiotensinogen-aldosterone system and superoxide and proinflammatory adipokines, such as: TNF- $\alpha$ and adipocyte fatty acid binding protein.

\section{Adipose tissue dysfunction, inflammation and cardiovascular disease}

The expansion and remodeling of adipose tissue in obesity causes big changes in both metabolic and vascular functions of this tissue. In both animals and humans with obesity, adipocyte hypertrophy causes local hypoxia, which in turn triggers infiltration of macrophages and other immune cells into adipose tissue. In many clinical and animal studies has been confirmed that inflammation and dysfunction of adipose tissue plays a major role in the pathogenesis of vascular endothelial dysfunction. It has been observed that in condition of obesity there is an increased macrophage infiltration and has a major expression of pro-inflammatory factors in subcutaneous abdominal fat. This is concluded to be associated with insulin resistance and systemic arterial dysfunction. In obesity, inflamed adipose tissue contributes to vascular dysfunction via multiple mechanisms. Data from both animal studies and clinical investigations have provided compelling evidence demonstrating that adipose tissue and blood vessels are associated, from early to adult life. These two organs communicate with each other by secreting a large number of paracrine and endocrine signals, which are actively involved in angiogenesis, adipogenesis, metabolism, and vascular homeostasis. ${ }^{21,23}$

\section{Adipose tissue dysfunction and obesity}

It has been established that obesity is associated with the appearance of a chronic, low inflammatory state, due to changes in function of adipocytes and macrophages. The term adipose tissue dysfunction is generally used for this state of hyper secretion of pro-atherogenic, pro-inflammatory and pro-diabetic adipocytokines which is accompanied by a decreased production of adiponectin. ${ }^{4}$ Obesity has a strong genetic predisposition, and results from an excess energy intake and too little energy expenditure. Obesity is associated with marked changes in the secretory function of adipocytes and macrophages, together with chronic inflammation and an increased risk to develop insulin resistance, diabetes, and vascular disease. Macrophages are more prevalent in adipose tissue of obese organisms and the macrophage quantity correlates with measures of insulin resistance. There are two types of macrophages which enter the adipose tissue- M1-macrophages (predominant in obesity secreting TNF-a and IL-6 thereby enhancing inflammation), and type M2-macrophages secreting anti-inflammatory cytokines such as IL-10, which has a function in tissue repair. Both macrophages and adipocytes are capable of accumulating lipids and secreting cytokines. It is established that the number of macrophages in adipose tissue is reduced after weight loss. ${ }^{4,25}$

Adipogenesis and angiogenesis are two closely related processes during adipose tissue enlargement and this is shown in animal studies and in vitro models. As adipocyte hypertrophy endures, local adipose tissue hypoxia may occur due to hypo-perfusion. This happens because the diameter of fat cells overgrows and the penetration of oxygen is reduced. Not only may the amount of fatty acids be relevant for mediating adipocyte function, but also the quality of the fatty acids. For example, dietary fish-oil, rich in long-chain unsaturated FFAs, increases activity and mRNA levels of FA oxidation enzymes in peroxisomes and mitochondria. It has been reported that polyunsaturated fatty acids reduce mortality and morbidity in patients who suffered from a myocardial infarction.

Leptin- a hormone made by fat cells which regulates the amount of fat stored in the body. In leptin-deficient humans and in animal models, leptin administration results in reduced body mass and decreased hyperphagia. Although obese subjects have high levels of leptin, their energy expenditure and appetite are not sufficiently regulated. Therefore it is concluded that there is a hypothalamic leptin resistance, which may be responsible for the persistent hunger and the difficulty to lose weight in obese subjects. It is known that insulin has similar properties as leptin in the hypothalamus so this may imply the existence of vicious circles of leptin resistance and insulin resistance which both lead to hunger and less energy expenditure. ${ }^{4}$

Most blood vessels are surrounded by adipose tissue. Perivascular adipose tissue (PVAT) is the adipose tissue surrounding blood vessels. PVAT is now considered a highly active endocrine organ that releases a variety of adipokines, inflammatory cytokines, and other factors which influence vascular tone in a paracrine way. Under physiological conditions, PVAT releases a number of vasoactive substances, such as adiponectin, angiotensin- $(1,11-12,15-16,22), \mathrm{H}_{2} \mathrm{O}_{2}$, leptin, and nitric oxide (NO). They elicit a beneficial anticontractile effect on vascular function and are essential for the maintenance of vascular resistance. It is now known that obesity triggers both structural and functional changes in PVAT which seem to be related to endothelial dysfunction and vascular damage. ${ }^{23}$ 
In 1991, Soltisand Cassis has demonstrated for the first time that PVAT reduced contractions to noradrenaline in rat aorta. Since then, an important number of adipocyte-derived factors with physiological and pathophysiological paracrine vasoactive effects have been identified. ${ }^{21}$ PVAT undergoes structural and functional changes in obesity. Thus in established obesity, PVAT loses its anticontractile properties by an increase of contractile, oxidative, and inflammatory factors, leading to endothelial dysfunction and vascular disease. Obesity is an independent risk factor for the development of endothelial dysfunction and vascular disease, hypertension, myocardial infarction, and stroke. The probability of suffering from vascular diseases is four times higher in obese (body mass index $>30 \mathrm{~kg} / \mathrm{m}^{2}$ ) than in normal-weight people (body mass index $\leq 25 \mathrm{~kg} / \mathrm{m}^{2}$ ). Adipose tissue acts as an endocrine organ by secreting various signaling cytokines, called adipokines, which affect energy metabolism, insulin sensitivity, inflammatory response, and blood flow. ${ }^{21}$

\section{Vascular dysfunction in obesity - Adipose tissue dysfunction and vascular diseases}

Atherosclerotic vascular disease may also be an important clinical result of adipose tissue dysfunction. Elevated blood pressure, low plasma HDL-c (high density lipoprotein cholesterol) and elevated TG are closely associated with abdominal obesity and can often be controlled by dietary changes and weight reduction. It is suggested that an activated renin-angiotensin-aldosterone system (RAS) and leptin are involved in obesity-associated hypertension by influencing the salt-fluid homeostasis and vascular tone. In obese people, plasma angiotensinogen (AGT) and renin concentrations are elevated and angiotensin converting enzyme (ACE) activity is increased. Angiotensin II may impair intracellular insulin leading to reduced glucose uptake. In obese organisms weight loss results in decreased leptin levels. Moreover in presence of hypertension, lower blood pressure resulted with a calorie restricted diet. The concept of leptin-provoked hypertension is based on the findings that leptinupregulates $\mathrm{Nap} / \mathrm{Kp}$-ATPase in the renal cortex and medulla. In the brain leptin leads to an increased sympathetic nerve activity directed to the kidneys and peripheral vasculature which leads to increased heart rate and elevated blood pressure levels in mice. In addition to leptin, renal sodium reabsorption is enhanced under insulin-resistant conditions and associated hyperinsulinaemia. ${ }^{4,26}$

\section{Adipocytokines and vascular disease}

A low plasma adiponectin concentration is a good representative of adipocyte dysfunction. Adiponectinis considered being an important causal link between dysfunctional adipocytes and the development of vascular diseases. In various populations of healthy people and high risk patients, low plasma levels of adiponectin are independent predictions of future vascular disease. ${ }^{4}$ It has been shown that obesity can lead to localized hypoxia. The reduction in blood flow in obese adipose tissue could limit the delivery of nutrients and thus to lead to insulin resistance. It can be posited that obesity enters a vicious cycle whereby endothelial cell dysfunction in the adipose tissue leads to metabolic dysfunction. It is reflected by adipokine dysregulation, adipocyte necrosis and inflammation. In turn, metabolic dysfunction promotes endothelial cell dysfunction, both in the adipose tissue and systemic circulation, putting further stress on adipocytes. However, it has been reported that obese mice receiving anti-angiogenic reagents have a reduced body weight and adipose mass, and show increased metabolic rates. ${ }^{24}$

\section{Discussion}

Increasing physical activity and weight reduction are two important lifestyle changes to reduce insulin resistance and visceral obesity. The most important thing is the decreasing the size of the adipocytes. It has been established that after 3 months of restricted caloric intake and increased exercise TNF-a, leptin, and IL-6 levels decreased. On the other hand anti-inflammatory cytokines (adiponectin and IL-10) were significantly increased in obese subjects with metabolic risk factors. Insulin sensitivity improved, even before weight reduction and adiponectin plasma levels increased suggesting that adipocyte function has improved. ${ }^{4}$

However, changes in lifestyle are difficult to achieve and to maintain in clinical practice, but are required for a constant improvement of a patients' metabolic profile. Some drugs may be of main interest of preventing further health complications. Statins have anti-inflammatory properties which may point towards an influence of statins on adipocyte function. Nevertheless, short term treatment with simvastatin alone or in combination did not result in changes in adiponectin, leptin, and resisting levels in healthy and obese patients with metabolic syndrome. ${ }^{4,27}$

\section{Conclusion}

The classical perception of adipose tissue as a storage depot of FFAs has now been replaced by the idea that adipose tissue is an active endocrine organ which plays a central role in lipid and glucose metabolism. It also produces a large number of hormones and cytokines involved in the development of metabolic syndrome, diabetes mellitus, and vascular diseases. Macrophages infiltrate adipose tissue when it expands. Thus the production of adipocytokines involved in glucose and lipid metabolism and in the inflammation increases. On the other hand the production of adiponectin decreases. Weight reduction and increasing physical activity are effective interventions for improving adipose tissue function. . $^{412,27}$

\section{Acknowledgements}

None.

\section{Conflict of interest}

The author declares no conflict of interest.

\section{References}

\section{WHO. Obesity and overweight. 2014.}

2. Beydoun MA, Beydoun HA, Wang Y. Obesity and central obesity as risk factors for incident dementia and its subtypes: a systematic review and meta-analysis. Obes Rev. 2008;9(3):204-218.

3. Bosello O, Zamboni M. Visceral obesity and metabolic syndrome. Obes Rev. 2000;1(1):47-56.

4. Hajer GR, van Haeften TW, Visseren FL. Adipose tissue dysfunction in obesity, diabetes, and vascular diseases. Eur Heart J. 2008;29(24):29592971.

5. Cole TJ, Freeman JV, Preece MA. Body mass index reference curves for the UK, 1990. Arch Dis Child. 1995;73(1):25-29.

6. Despres JP. Is visceral obesity the cause of the metabolic syndrome? Ann Med. 2006;38(1):52-63.

7. Lean ME, Han TS, Morrison CE. Waist circumference as a measure for indicating need for weight management. BMJ. 1995;311(6998):158-161. 
8. Dennis KE, Goldberg AP. Differential effects of body fatness and body fat distribution on risk factors for cardiovascular disease in women. Arterioscler Thromb. 1993;13(10):1487-1494.

9. Despres JP, Couillard C, Gagnon J, et al. Race, visceral adipose tissue, plasma lipids, and lipoprotein lipase activity in men and women:the Health, Risk Factors, Exercise Training, and Genetics (HERITAGE) family study. Arterioscler Thromb Vasc Biol. 2000;20(8):1932-1938.

10. Dietz WH. Birth weight, socioeconomic class, and adult adiposity among African Americans. Am J Clin Nutr. 2000;72(2):335-336.

11. WHO. Global status report on noncommunicable diseases 2010. 2011.

12. Cecchini M, Sassi F, Lauer JA, et al. Tackling unhealthy diets, physica inactivity, and obesity: health effects and cost-effectiveness. Lancet 2010;376(9754):1775-1784.

13. Misra A, Wasir JS, Vikram NK. Waist circumference criteria for the diagnosis of abdominal obesity are not applicable uniformly to all populations and ethnic groups. Nutrition. 2005;21(9):969-976.

14. Must A, Jaques PF, Dallal GE, et al. Long term morbidity and mortality of overweight adolescents:a follow-up of the Harvard growth study of 1922 to 1935. N Engl J Med. 1992;327(19):1350-1355.

15. WHO. Obesity: preventing and managing the global epidemic: report of a WHO Consultation. World Health Organ Tech Rep Ser. 2000;894(ixii):1-253.

16. Van Gaal LF, Mertens IL, De Block CE. Mechanisms linking obesity with cardiovascular disease. Nature. 2006;444(7121):875-880.

17. Alberti KG, Zimmet P, Shaw J. The metabolic syndrome-a new worldwide definition. Lancet. 2005;366(9491):1059-1062.
18. Reilly JJ, Wilson ML, Summerbell CD, et al. Obesity: diagnosis, prevention, and treatment; evidence based answers to common questions. Arch Dis Child. 2002;86(6):392-394.

19. Armstrong J, Reilly JJ, Child Health Information Team. Breastfeeding and lowering the risk of childhood obesity. Lancet. 2002;359(9322):20032004.

20. Despres JP, Lemieux I. Abdominal obesity and metabolic syndrome Nature. 2006;444(7121):881-887.

21. Fernandez-Alfonso MS, Gil-Ortega M, Garcia-Prieto CF, et al. Mechanisms of Perivascular Adipose Tissue Dysfunction in Obesity. Int J Endocrinol. 2013;2013:402053.

22. Isomaa B, Almgren P, Tuomi $\mathrm{T}$, et al. Cardiovascular morbidity and mortality associated with the metabolic syndrome. Diabetes Care. 2001;24(4):683-689.

23. $\mathrm{Gu} \mathrm{P}, \mathrm{Xu} \mathrm{A}$. Interplay between adipose tissue and blood vessels in obesity and vascular dysfunction. Rev Endocr Metab Disord. 2013;14(1):49-58.

24. Ouchi N, Parker JL, Lugus JJ, et al. Adipokines in inflammation and metabolic disease. Nat Rev Immunol. 2011;11(2):85-97.

25. Parsons TJ, Power C, Logan S, et al. Childhood predictors of adult obesity: systematic review. Int J Obes Relat Metab Disord. 1999;23(suppl 8):S1-S107.

26. Reilly JJ, Methven E, McDowell ZC, et al. Health consequences of obesity. Arch Dis Child. 2003;88(9):748-752.

27. Whitaker RC. Obesity prevention in primary care: four behaviors to target. Arch Pediatr Adolesc Med. 2003;157(8):725-727. 